\title{
AVALIAÇÃO DE MÉTODOS PARA MONITORAR POPULAÇÕES DE ARTRÓPODES EM GRANJA AVÍCOLA, EM PELOTAS, RIO GRANDE DO SUL, BRASIL
}

\section{D.M. Pinto, P.B. Ribeiro, E. Bernardi}

Universidade Federal de Pelotas, Departamento de Microbiologia e Parasitologia, CP 354, CEP 96010-900, Pelotas, RS, Brasil.

RESUMO

A comunidade de artrópodes encontrados em granjas tem apresentado grande importância médica e veterinária, pois são vetores de várias doenças, causando problemas sanitários e econômicos. N este trabalho foi realizada a avaliação comparativa de métodos de monitoramento de artrópodes em granjaavícola. As aval iações foramfeitas em um período de 12 meses, em gal pão de poedeirasdoConjunto A grotécnicoViscondedaGraça, emPelotas, RS, Brasil, utilizandoarmadilhas do tipo "tubo" ou de A rends etipo "sanduíche". A armadilha do tipo sanduíche mostrou-se mais eficientenacapturadosartrópodes, sendocapturadasasespéciesA I phitobius diaperinus (Coleoptera), Carcinops troglodytes (Coleoptera), Euspilotus rubriculus (Coleoptera), G nathocerus cornutus (Coleoptera), M ezium americanum (Coleoptera), Somotrichus unifasciatus (Coleoptera), Euborellia annulipes (Dermaptera), D rosophila repleta (Diptera) eW ithius piger (Pseudoscorpionida).

PALAVRAS-CHAVE: Armadilhas, ecossistema, vetores, comunidade, esterco de aves.

\section{ABSTRACT}

EVALUATION OFTHEMETHODSFORMONITORING POPULATIONSOFARTHROPODSON POULTRY FARMSIN PELOTAS, RIO GRANDE DO SUL, BRAZIL. The community of arthropods found on farms is currently of great medical and veterinary concern, because they are vectors of various diseases, causing sanitary and economic problems. In this study, arthropod monitoring methodswerecomparatively evaluated on a poultry farm. Theevaluationsweremadeover aperiod of 12 months, in a hen house of the Conjunto A grotécnico Visconde da Graça, in Pelotas, RS, Brazil, using "tube-type" or Arends traps and "sandwich-type" traps. The "sandwich-type" trap proved more efficient in the capture of the arthropods, the species captured being A Iphitobius diaperinus (Coleoptera), Carcinops troglodytes (Coleoptera), Euspilotus rubriculus (Coleoptera), Gnathocerus cornutus (Coleoptera), M ezium americanum (Coleoptera), Somotrichus unifasciatus (Coleoptera), Euborellia annulipes (Dermaptera), D rosophila repleta (Diptera) and W ithius piger (Pseudoscorpionida).

KEY WORDS: Traps, ecosystem, vectors, community, poultry manure.

\section{INTRODUÇÃO}

A necessidade de produzir quantidades cada vez maiores dealimentoslevou ohomemaprocurar formas de produção intensivas. Com esse propósito, uma das alternativasparaumamaior produção dealimentos de origem animal foi a criação intensiva através de confinamento.Tal medidaaumentouaconcentraçãode excretas deanimais, criando, nesteslocais, ecossistemas artificiais propíciosà proliferação deartrópodes. Dessa forma al gumas espécies de artrópodes sinantrópicos passaram a ocorrer em grande número e por al gumas delas serem vetores de patógenos, apresentam grande importância médica eveterinária (FrancISCO, 1996).

Portanto, o estudo das espécies que ocorrem em ambientes modificados pelo homem assume impor- tância não só ecológica, pois a associação destas espécies à veiculação dos mais diversos organismos patogênicos, serevestetambém, deinteressesanitário (MASCARINI, 1995).

A diversidadedeartrópodesencontradaemesterco acumulado, nos locais de criação de aves domésticas, é muito grande; e esses artrópodes são principalmente, espéciesdecoleópteros, dípteroscidorrafos e ácaros (AxTELL; ArendS, 1990).

Alguns trabalhos vêm sendo realizados com o objetivo de conhecer melhor o agroecossistema das granjas avícolas e indicam algumas espécies de coleópteros das famílias Histeridae e Staphylinidae (Pfeiffer; Axtell, 1980; Axtell, 1986a; 1986b; Axtell; ARENDS, 1990; Brunoetal ., 1993) eácarosM acrochelidae eUropodidae(H ALL, 1985; A xTELL, 1986a; 1986b;GEDEN; 
Axtell, 1988; Axtell; Arends, 1990) como principais predadoresdeovoselarvas dedípterosnessasgranjas.

O monitoramento das populações de artrópodes emaviárioséum procedimento quedeveser adotado dentro do programa de manejo, independente da estratégiautilizada para o controle(Lefferet al.,2001; HICKLE, 1997). Entretanto, ausência de um método prático quantitativo para amostragem de população de insetos e ácaros, tem impedido a obtenção de informações necessárias para serem utilizadas em programas de controle biológico dês artrópodes em aviários (H ARDING; BISSEL, 1958; SIMCo et al., 1967).

Tendo em vista a necessidade de determinar métodos para o monitoramento e estudo das espécies que ocorrem em um ecossistemaartificial etambém devido apouca pesquisa paraaregiãosul doBrasil queaborde tal assunto, foi desenvolvido estetrabal ho comoobjetivo de identificar e determinar o melhor método para monitorar artrópodesemgranjaavícolaem Pelotas, RS.

\section{MATERIAL E MÉTODOS}

O trabal ho foi realizado na granja do Conjunto Agrotécnico ViscondedaGraça(CAVG), pertencente àUniversidadeFederal dePelotas, em Pelotas, RS, no período de abril de 2002 a março de 2003.

Cada gal pão apresenta uma área de $245 \mathrm{~m}^{2}(20 \mathrm{~m}$ de comprimento $\times 12,25 \mathrm{~m}$ de largura) e capacidade para abrigar de 1.200 a 3.400 aves, dependendo do tipo de criação.

O gal pão de poedeiras em cama com maraval has apresenta um puleiro central ( $14 \mathrm{~m}$ decomprimento $\mathrm{x}$ 10 mdelargurax 0,6 m dealtura) formado por muretas deconcreto e coberto por um estrado de madeira com tela. Sobre esse, estão suspensos os bebedouros e os comedouros. No seu interior, as fezes das aves ficam retidas durantetodoo período depermanênciadolote, funcionando, destaforma, como refúgioepreservação da diversidadedeartrópodes. As saves não têmacesso às fezes. A área de circulação ao redor do puleiro apresentaumacamadademaraval hascomcercade10 cmdealtura, repostasemprequenecessário. Osninhos encontram-sefixados ao longo detrês das paredes do gal pão ea meio metro do chão.

A coletafoi realizadaatravés dedoismétodos, com o objetivo de verificar a diversidade de artrópodes capturados, conforme a descrição abaixo:

Armadilha do tipo "sanduíche"; Armadilha do tipo "tubo".

- Armadilha do tipo "tubo" ou deArends (SAFRIT; AxTELL, 1984)-consisteemumtubo depolivinilcloreto (PVC) de3,8cm dediâmetro por $23 \mathrm{~cm}$ decomprimento, contendo em seu interior papel corrugado, colocado de forma que as ondulações fiquem dispostas em sentido longitudinal ao tubo.
- Armadilha do tipo "sanduíche" (SAFrIt; Axtell, 1984) - consiste em caixas de madeira de $20 \mathrm{~cm}$ de comprimento por $15 \mathrm{~cm}$ delargurae $8 \mathrm{~cm}$ dealtura, com tampa eduas aberturas de $1 \mathrm{~cm}$ no sentido longitudinal, junto a base da caixa, para facilitar a entrada do coleóptero. No interior das armadilhas foi colocado papel corrugado, de modo que preenchesse a caixa.

Foram instaladas 16 armadilhas, em grupos de quatro, em quatro locais diferentes do galpão, eqüidistantes $30 \mathrm{~cm}$ umas das outras. Cada grupo estava composto por duas armadilhas do tipo "sanduíche" e duas armadilhas do tipo "tubo".

Foramfeitascoletas semanais, ondeo conteúdo de cada armadilha era colocado em um pote plástico, devidamenteidentificado, com o auxilio de um funil de alumínio. Os papéis corrugados das armadilhas eram substituídos por papéis novos e os artrópodes capturados eram levados para o laboratório onde eram feitas triagens, identificação e contagem dos mesmos. O material encontrado, em cada armadilha, bem como a data e as observações pertinentes foram registradosemfichacontrole. A pósesseprocedimento a artropodofauna era colocada em potes de vidro, devidamente identificados, com álcool para conservação. Os resultados obtidos foram submetidos a análise da variação e teste de "F", utilizando-se o programa estatístico SANEST.

\section{RESULTADOSE DISCUSSÃO}

Durante o período experimental, foram capturados 45.764artrópodespertencentesàs classes Insecta eA rachnida. A classel nsectarepresentou 96,64\%, do total de artrópodes capturados (Tabela 1).

Ao comparar os métodos deamostragem para as classesInsectaeA rachnida, verificou-seumadiferença em relação à freqüência dos espécimens coletados (Tabela 1). N as col etas realizadas utilizando a armadilha do tipo sanduíche, foram encontrados 42.600 dos 44.228 insetos capturados, ou seja, $96,32 \%$.

A maior freqüênciadecoleta daclasseA rachnida, tambémocorreu quando foi utilizadaa armadilhado tipo sanduíche, tendo capturado 1.189 dos 1.536 espécimens, ou seja, $77,41 \%$.

A classelnsecta esteverepresentadapelasordens Coleoptera, Dermaptera e Diptera, enquanto que a classe Arachnida esteve representada pela ordem Pseudoscorpionida. A ordem Coleoptera foi a mais representativa com $96,03 \%$ dos insetos capturados (Tabela 1).

Os coleópteros encontrados foram Alphitobius diaperinus (Panzer, 1797) (Tenebrionidae); Carcinops troglodytes (Paykull, 1811) (Histeridae); Euspilotus rubriculus (Marseul, 1855) (Coleoptera: Histeridae); Gnathocerus cornutus (Fabricius, 1798) (Tenebrionidae); 
M ezium americanum (Linnaeus, 1758) (Ptinidae); Somotrichus unifasciatus (Dejean, 1792) (Carabidae), da ordem Dermaptera foi encontrada Euborellia annulipes (Lucas, 1842) (Anisolabididae), da ordem Diptera foi encontradaD rosophila repleta (Wollaston, 1858) (Drosophilidae) eda ordem Pseudoscopionida W ithius piger (Simon, 1878) (Withiidae) (Tabela 1).

Dentre os artrópodes coletados, a ordem Coleoptera, apresentou o maior número de espécies capturadas (96,03\%), havendo predomínio da família Tenebrionidae $(94,70 \%)$ (Tabela 1). Esses resultados diferem dos obtidos por Bıсно (2001), em granja avícola em Pelotas, RS, que encontrou, na mesma granja, a família Histeridae como a mais abundante (53,47\%) seguidapelaTenebrionidaecom $31,09 \%$ das 10 famílias encontradas, isso, provavel mentedevido ao método utilizado, coleta de fezes acumuladas. A A GEnSEn (1988) e Bruno et al. (1993), em aviários, de poedeiras criadas em gaiolas, em diversos municípios de São Paulo, também encontraram a família Histeridae como a mais abundante, 36,12\% e 39\%, respectivamente. Entretanto, Pfeiffer; Axtell (1980) citaram a família Tenebrionidae como a mais abundanteem 3regiões amostradas da Carolina do N orte, juntamente com a família Histeridae.

Segundo BALDuF (1935), histerídeossão comumente encontrados em plantas em decomposição, excremento e carne putrefata. Wenzel (1961), apud PECK (1968) afirmou quetodasaslarvasdehisterídeos são predadoras e GeETHA BAI; SANKARAN (1977) consideraramoshisterídeoscomo eficientespredadoresde muscóides, sendo bastante promissores no controle biológico de moscas, isto talvez explique a baixa capturadestafamília, devido aosmétodosutilizados.

ComopodeseobservarnaTabela1,A . diaperinusfoi aespéciemaiscapturadaentreoscoleópteros(89,65\%). Esteresultadoésemel hanteao encontrado porA AGESEN (1988) que observou uma presença bastante acentuada, principalmente de larvas desse coleóptero, em excremento deaves, em aviários de Bastos, SP.

A diversidade de artrópodes encontrada, no presentetrabalho, foi inferior aobservada por $\mathrm{BICHO}$ (2001), isto provavelmenteseexpliquepeladiferença dos métodos utilizados, uma vez que o autor utilizou fezes de aves em diferentes estágios de decomposição, além da armadilha tipo tubo, para avaliar a população de artrópodes em granja avícola.

Tabela 1 - Freqüência de artrópodes capturados em gal pão de aves poedeiras, no período de abril de 2002 a março de 2003, em Pel otas, RS.

\begin{tabular}{|c|c|c|c|c|c|c|c|c|}
\hline \multirow[t]{2}{*}{ Espécimes } & \multicolumn{3}{|c|}{ Armadilha tipo sanduíche } & \multicolumn{3}{|c|}{ Armadilha tipo tubo } & \multicolumn{2}{|c|}{ Total } \\
\hline & $\begin{array}{c}\text { Fr. abs. } \\
\left(n^{\circ}\right)\end{array}$ & $\begin{array}{c}\text { Fr. rel. } \\
(\%)\end{array}$ & $\begin{array}{l}\text { Média } \\
\text { captura }\end{array}$ & $\begin{array}{l}\text { Fr. abs. } \\
\left(n^{\circ}\right)\end{array}$ & $\begin{array}{l}\text { Fr. rel. } \\
\text { (\%) }\end{array}$ & $\begin{array}{l}\text { Média } \\
\text { captura }\end{array}$ & $\begin{array}{c}\text { Fr. abs. } \\
\left(n^{\circ}\right)\end{array}$ & $\begin{array}{c}\text { Fr.rel. } \\
(\%)\end{array}$ \\
\hline Insecta & 4.2600 & 96,32 & & 1.628 & 3,68 & & & \\
\hline Coleoptera & 4.2379 & 99,48 & & 1.566 & 96,20 & & & \\
\hline Tenebrionidae & & & & & & & 41.617 & 94,70 \\
\hline Alphitobius diaperinus (larvas) & & & $6,62 a$ & & & $0,73 b$ & & \\
\hline Alphitobius diaperinus (adultos) & & & $193,72 a$ & & & $6,23 b$ & & \\
\hline Gnathocerus cornutus & & & $1,89 a$ & & & $0,64 b$ & & \\
\hline Histeridae & & & & & & & 795 & 1,81 \\
\hline Carcinops troglodytes & & & $2,82 a$ & & & $0,12 \mathrm{~b}$ & & \\
\hline Euspilotus rubriculus & & & $1,27 a$ & & & $0,14 b$ & & \\
\hline Ptinidae & & & & & & & 367 & 0,84 \\
\hline M ezium americanum & & & $2,15 a$ & & & $0,07 b$ & & \\
\hline Carabidae & & & & & & & 1166 & 2,65 \\
\hline Somotrichus unifasciatus & & & $5,74 a$ & & & $0,24 b$ & & \\
\hline Dermaptera & 205 & 0,48 & & 60 & 3,68 & & & \\
\hline A nisolabididae & & & & & & & 265 & 4,16 \\
\hline Euborellia annulipes & & & $1,47 a$ & & & $0,36 \mathrm{~b}$ & & \\
\hline Diptera & 17 & 0,04 & & 2 & 0,12 & & & \\
\hline Drosophilidae & & & & & & & 19 & 0,16 \\
\hline Drosophila repleta & & & $0,11 a$ & & & $0,01 a$ & & \\
\hline Arachnida & 1.189 & 77,41 & & 347 & 22,59 & & & \\
\hline Pseudoscorpionida & & & & & & & 1.536 & 3,36 \\
\hline Whitius piger & & & 10,06a & & & $2,92 \mathrm{~b}$ & & \\
\hline
\end{tabular}

Médias seguidas de letras distintas, nas linhas, diferem significativamente entre si pelo teste de Duncan. 
$\mathrm{Na}$ classe Insecta, registrou-se a presença das ordens Dermaptera, Coleoptera eDiptera. Conforme aTabela 1, o maior número deinsetoscapturados, nos 2 métodos utilizados, pertence à ordem Coleoptera com 99,48\% naarmadilhatipo "sanduíche" e96,20\% na armadilha tipo "tubo".

Em todos os dados obtidos na comparação das armadilhas, a do tipo "sanduíche" mostrou-sesuperior à do tipo tubo, em relação à captura e monitoramento deartrópodes, principalmenteadultos de A diaperinus em granja de aves, diferindo de
FranCISCO (1996) queconsiderou aarmadilhade "tubo" ou A rends como o melhor método para amostragem de adultos de A . diaperinus.

BıcHo (2001), ao comparar diferentes métodos de coletas de artrópodes em aviário de poedeiras concluiu que a armadilha tipo "tubo" foi responsável, juntamente com fezes acumuladas, pelas maiores médias decaptura dearacnídeos. Porém, no presente trabal ho, a armadilha tipo "sanduíche" foi a responsável pelas maiores médias decaptura, quando comparada com a tipo tubo.

Tabel a 2 - A nálise devariação do número de indivíduos, de cada espécie deArtrópodes, capturados em granja avícola, em Pelotas, RS, no período de abril de 2002 a março de 2003, que indicaram significância, pelo teste F, para os meses do ano, para os métodos de col eta e para a interação desses fatores.

\begin{tabular}{lccc}
\hline \multirow{2}{*}{ Espécies } & \multicolumn{3}{c}{ Causas da variação } \\
\cline { 2 - 4 } & Meses & Métodos & MesesxMétodos \\
\hline Alphitobius diaperinus (larvas) & $8,03^{*}$ & $37,04^{*}$ & $3,76^{*}$ \\
Alphitobius diaperinus (adultos) & $53,84^{*}$ & $49,54^{*}$ & $28,10^{*}$ \\
Carcinops troglodytes & $12,76^{*}$ & $17,67^{*}$ & $9,90^{*}$ \\
Euborellia annulipes & $11,75^{*}$ & $17,13^{*}$ & $0,99^{* * *}$ \\
Euspilotus rubriculus & $10,43^{*}$ & $22,85^{*}$ & $5,55^{*}$ \\
Gnathocerus cornutus & $33,60^{*}$ & $12,66^{*}$ & $4,4^{*}$ \\
M ezium americanum & $25,59^{*}$ & $50,99^{*}$ & $22,09^{*}$ \\
Somotrichus unifasciatus & $40,09^{*}$ & $46,52^{*}$ & $24,96^{*}$ \\
Withius piger & $14,77^{*}$ & $144,00^{*}$ & $1,93^{*}$ \\
\hline
\end{tabular}

(*) nível de significância a 5\% e (***) ausência de significância.

Tabela 3- Ocorrência mensal de Col eoptera, Dermaptera, Diptera ePseudoscorpionida capturados em gal pão deaves poedeiras, no período de abril de 2002 a março de 2003, em Pelotas, RS.

\begin{tabular}{|c|c|c|c|c|c|c|c|c|c|c|c|c|c|c|c|}
\hline \multirow[b]{2}{*}{ Ordem } & \multirow[b]{2}{*}{ Família } & \multirow[b]{2}{*}{ Espécie } & \multicolumn{13}{|c|}{ Meses (2002/ 2003) } \\
\hline & & & $A$ & $M$ & $J$ & $J$ & $A$ & $\mathrm{~S}$ & $\mathrm{O}$ & $\mathrm{N}$ & $\mathrm{D}$ & $J$ & $\mathrm{~F}$ & $M$ & A \\
\hline \multirow[t]{6}{*}{ Coleoptera } & Tenebrionidae & $\begin{array}{l}\text { Alphitobius } \\
\text { diaperinus }\end{array}$ & $x$ & $x$ & $x$ & $x$ & $x$ & $x$ & $x$ & $x$ & $x$ & $x$ & $x$ & $x$ & $x$ \\
\hline & & $\begin{array}{l}\text { Gnatocerus } \\
\text { cornutus }\end{array}$ & & & $x$ & & $x$ & $x$ & $x$ & $x$ & $x$ & $x$ & $x$ & $x$ & $x$ \\
\hline & Histeridae & $\begin{array}{l}\text { Carcinops } \\
\text { troglodytes }\end{array}$ & & $x$ & $x$ & & $x$ & $x$ & $x$ & $x$ & $x$ & $x$ & $x$ & $x$ & $x$ \\
\hline & & $\begin{array}{l}\text { Euspilotus } \\
\text { rubriculus }\end{array}$ & & $x$ & & & & $x$ & $x$ & $x$ & $x$ & $x$ & $x$ & $x$ & $x$ \\
\hline & Ptinidae & $\begin{array}{l}\text { M ezium } \\
\text { americanum }\end{array}$ & & & $x$ & $x$ & & $x$ & $x$ & $x$ & $x$ & $x$ & $x$ & $x$ & $x$ \\
\hline & Carabidae & $\begin{array}{l}\text { Somotrichus } \\
\text { unifasciatus }\end{array}$ & & $x$ & & $x$ & $x$ & $x$ & $x$ & $x$ & $x$ & $x$ & $x$ & $x$ & $x$ \\
\hline Dermaptera & Anisolabididae & $\begin{array}{l}\text { Euborellia } \\
\text { annulipes }\end{array}$ & $x$ & $x$ & $x$ & $x$ & $x$ & $x$ & $x$ & $x$ & $x$ & $x$ & $x$ & $x$ & $x$ \\
\hline Diptera & Drosophilidae & $\begin{array}{l}\text { Drosophila } \\
\text { repleta }\end{array}$ & & & $x$ & $x$ & & $x$ & $x$ & & & & & & \\
\hline Pseudoscorpionida & & $\begin{array}{l}\text { Whitius } \\
\text { piger }\end{array}$ & $x$ & $x$ & $x$ & $x$ & $x$ & $x$ & $x$ & $x$ & $x$ & $x$ & $x$ & $x$ & $x$ \\
\hline
\end{tabular}


A análise da variação foi feita para cada espécie, sendo que para $A$. diaperinus essa análise foi feita larvas e para adultos separadamente.

Então, a análisedavariação individual, dasespé cies capturadas indicou significância, pelo teste $F$, para os meses do ano, métodos de coleta e para a interação dessesfatorescom nível designificânciade $5 \%$, como pode ser observado na Tabela 2 .

A penas $D$. repleta, apresentou ausência de significância, pelo testeF, isso provavel mentedevido ao pequeno número de espécimes capturadas pelas armadilhas tipo "tubo" e tipo "sanduíche".

$O$ fato de, entre os dípteros, apenas a espécie $D$. repleta ter sido capturadanesteexperimento, diferedo obtido por BICHo (2001) eA AGESEN (1998), queutilizando fezes acumuladas para obtenção de artrópodes, encontraram várias espécies de dípteros, devido ao método utilizado.

Os resultados do teste de Duncan, ao nível de $5 \%$ deprobabilidade, apresentados naTabela 1, revelam queaarmadilhadotipo "sanduíche" foi ométodoque apresentou maior média de captura da maioria das espécies, diferindo significativamente da armadilha tipo “tubo" , comexceção apenasemrelaçãoàcaptura daD . repleta, ondeotestedeF, nãoindicou significância.

A Tabela3ilustraaocorrênciamensal deDermaptera, Coleoptera, DipteraePseudoscorpionida. N elapodese observar que, nos 12 meses decoleta, estiveram presentes os seguintes insetos: A lphitobius diaperinus (larvase adultos) (Coleoptera:Tenebrionidae), Euborelliaannulipes (Dermaptera: A nisolabididae) e o arachnideo W ithius piger (Pseudoscorpionida: Withiidae).

\section{CONCLUSÃO}

- A armadilha tipo sanduícheémais eficaz quea tipo tubo, para a captura de insetos e aracnídeos.

- Foram encontrados os seguintes coleópteros: A. diaperinus, C. troglodytes, E. rubriculus, G. cornutus, M . americanum eS. unifasciatus.

\section{REFERÊNCIAS}

A AGESEN, T.L.A rtrópodesassociadosa excrementosem aviários . 1988. 38p. Dissertação (Mestrado) -EscolaSuperior de A gricultura Luiz de Queiroz, Piracicaba, 1988.

AxteLL, R.C. Fly control in confined livestock and poultry production. Greensboro: CIBA-GEICY Corporation, 1986a. 59p. (Technical Monograph).

AxTELL, R.C. Fly management in poultry production: cultural, biological, and chemical. Poultry Science, v.65, p.657-667, 1986b.

AxtelL, R.C.; Arends, J.J. Ecology and management of arthropod pests of poultry. Annual Review of Entomology, v.35, p.101-126, 1990.
Bal, M.G.; Sankaran, T. Parasites, predators and other arthropods associated withM usca domestica andother flies breeding in bovine manure. Entomophaga, v.22, n.2, p.163-167, 1977.

BALDUF, W.V. The bionomics of entomophagous coleoptera. St. Louis: John S. Swift, 1935. v.1.

Bıcнo, C.L. Comunidadedeartrópodes eflutuação populacional circanual de dípteros e coleópteros, em granja avícola, em Pelotas - RS. 2001. 110p. Tese (Doutorado) - Universidade Federal do Paraná, Curitiba, 2001.

Bruno, T.V.; Gumarães, J.H.; Santos, A.M.; Tuccl, E. C. Moscas sinantrópicas (Diptera) e seus predadores que se criam em esterco de aves poedei ras confinadas, no estado de São Paulo, Brasil. R evista Brasileira de Entomologia, v.37, n.3, p.577-590, 1993.

Francisco, O. A Iphitobius diaperinus ( $\mathrm{P}$ anzer) (Coleoptera: Tenebrionidae) associado a esterco em granjas de aves poedei ras: fenol ogia, estrutura etária eparasitismo. 1996. 116p. Dissertação (M estrado) - Universidade Estadual de Campinas, Campinas, 1996.

Geden, C.J.; Axtell, R.C. Predation by Carcinops pumilio (Coleoptera: Histeridae) and M acrocheles muscadomesticae (A carina: Macrochelidae) on thehouse fly (Diptera: Muscidae): functional response, effects of temperature, and availability of alternative prey. Environmental Entomology, v.17, n.4, p.739-744, 1988.

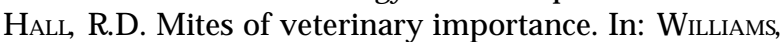
R.E.;HALL,R.D.;B ROCE,A.B.;ScholL,P.Livestockentomology. New York: Willey Interscience, 1985. p.151-181.

H ARDING JUNIOR, W.C.; BISSEL, T.L. Lesser mealworm in a brooder house. Journal of Economic Entomology, v.51, n.1, p.112, 1958.

HICKLE, L.A. Integrated pest management in poultry houses. Poultry Digest, v.56, p.1-23, 1997.

LefFer, A.M.C.; L azZARI ,F.A.;L aZzarI, S.M.N.; A lmeida,L.M. Controle do cascudinho. A vicultura Industrial, v.91, p 22-25, 2001.

Mascarinı, L.M. A spéctos biológicos de M uscina stabulans (Fallén, 1817) em condições de laboratório. 1995. 68p. Dissertação (Mestrado) - Universidade Estadual de Campinas, Campinas, 1995.

PECK, J.H. The potential role of arthropod predators in the integrated control of Diptera developing in poultry droppings. (Masters Thesis). Berkeley: University of California, 1968. 129p.

Pfeiffer, D.G.; AxtelL, R.C. Coleoptera of poultry manure in caged-layer housesin N orth Carolina.Environmental Entomology, v.9, p.21-28, 1980.

SAFRIT, R.D.; A xTELL, R.C.Eval uationsof sampling methods for darkling beetles (Alphitobius diaperinus) in the litter of turkey and broiler houses. P oultry Science, v.63, n.12,p.2368-2375, 1984.

Simco, J.S.; Everett, R.; LANCAster, J.L. Preliminary studies on control of lesser mealworm in broiler houses. A rkansas Farm Research, v.15, n.6, p.8, 1967.

Recebido em 3/ 11/ 05

A ceito em 13/ 4/ 07 